Document downloaded from:

http://hdl.handle.net/10251/83533

This paper must be cited as:

Pacheco-Paramo, DF.; Akyildiz, IF.; Casares Giner, V. (2016). Local Anchor Based Location Management Schemes for Small Cells in HetNets. IEEE Transactions on Mobile Computing. 15(4):883-894. doi:10.1109/TMC.2015.2431717.

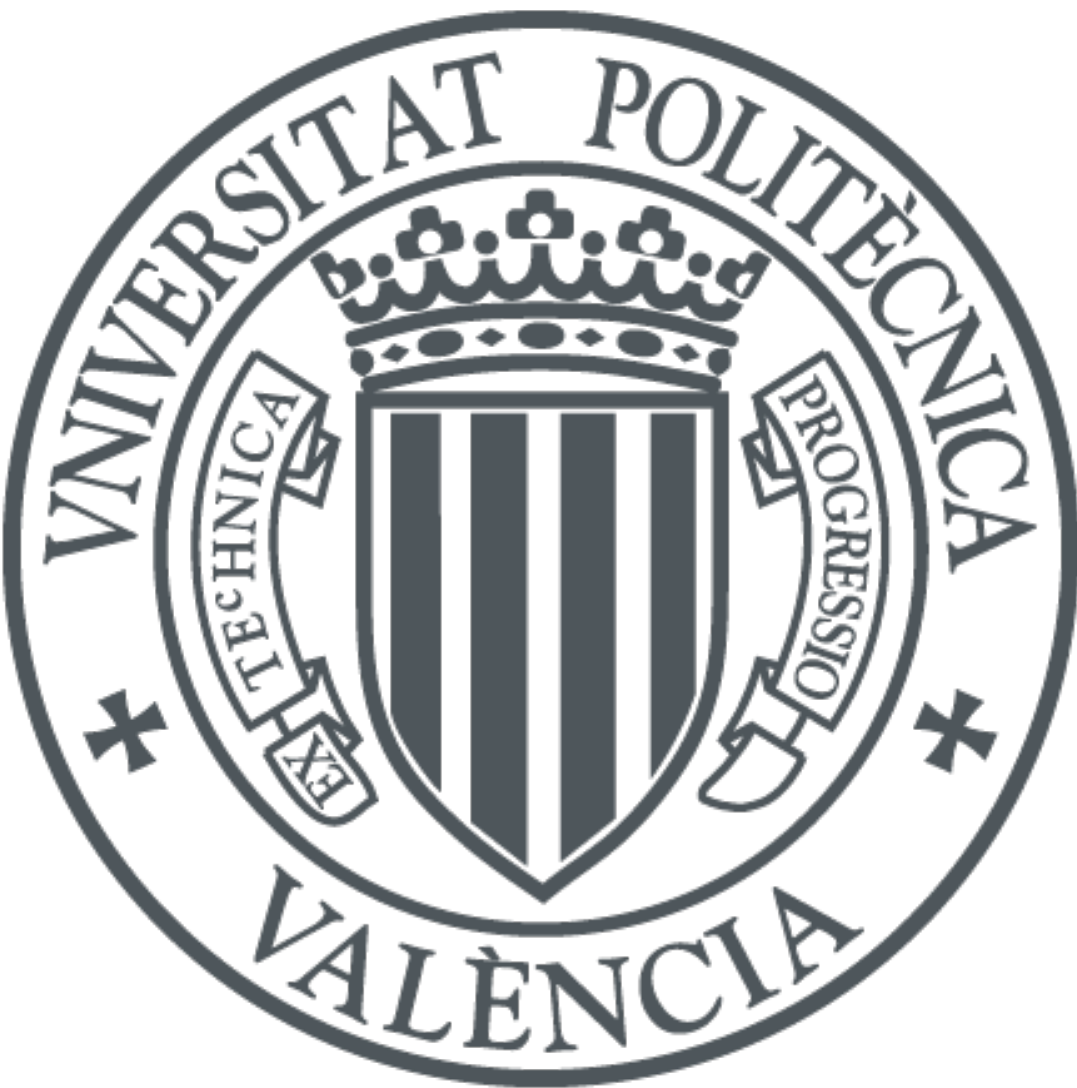

The final publication is available at

http://dx.doi.org/10.1109/TMC.2015.2431717

Copyright Institute of Electrical and Electronics Engineers (IEEE)

Additional Information

(c) 2015 IEEE. Personal use of this material is permitted. Permission from IEEE must be obtained for all other users, including reprinting/ republishing this material for advertising or promotional purposes, creating new collective works for resale or redistribution to servers or lists, or reuse of any copyrighted components of this work in other works. 


\title{
Local Anchor Based Location Management Schemes for Small Cells in HetNets
}

\author{
Diego Pacheco-Paramo, lan F. Akyildiz, Fellow, IEEE, \\ and Vicente Casares-Giner, Member, IEEE
}

\section{APPENDIX A \\ Discrete Markovian Arrival Process}

Let us formulate a Discrete Markovian Arrival Process (D-MAP) with the stochastic matrix $\mathbf{P}$ [32], in which, $\mathbf{D}_{0}$ and $\mathbf{D}_{1}$ are of size $N_{c} \times N_{c}$ with $N_{c}=S_{T A} \cdot S_{T A L}$ :

$$
\mathbf{P}=\left[\begin{array}{ccccc}
\mathbf{D}_{0} & \mathbf{D}_{1} & \mathbf{0} & \mathbf{0} & \ldots \\
\mathbf{0} & \mathbf{D}_{0} & \mathbf{D}_{1} & \mathbf{0} & \ldots \\
\mathbf{0} & \mathbf{0} & \mathbf{D}_{0} & \mathbf{D}_{1} & \ldots \\
\vdots & \vdots & \vdots & \vdots & \ddots
\end{array}\right]
$$

The probabilities in $\mathbf{D}_{0}$ and $\mathbf{D}_{1}$ have the following meaning. Suppose that, at an arbitrary epoch, the system is in state $i$, i.e., in SC $i$. At the end of the sojourn time in state $i$ a labeled or unlabeled transition towards state $j$ occurs with probability, respectively $D_{1 ; i, j}$ or $D_{0 ; i, j},\left(i, j \in 0,1,2, \ldots, N_{c}-1\right)$. A labeled transition is identified when the UE exits the Tracking Area List (TAL). An unlabeled transition is identified when the UE moves among SCs that belong to the same TAL. We assume that the sojourn time in any SC is characterized by the density function $f_{m}(t)$, with Laplace Transform (LT) $f_{m}^{*}(s)$, which means that we are in presence of a Semi-Markov process. The stochastic matrix associated with the underlying embedded Discrete Time Markov Chain (DTMC) is:

$$
\boldsymbol{\Pi}=\mathbf{D}_{0}+\mathbf{D}_{1}
$$

- D. Pacheco-Paramo was with Broadband Wireless Networking Lab, Georgia Institute of Technology, Atlanta, GA, 30332, USA. Now he and V. Casares-Giner are with Universitat Politècnica de València, Camino de Vera, s/n, 46022, Valencia, Spain. e-mail:(diepacpa@posgrado.upv.es and vcasares@dcom.upv.es).

- I. Akyildiz is with the Broadband and Wireless Networking Laboratory, School of Electrical and Computer Enoineering, Georgia Institute of Technology, Atlanta, GA 30332 USA. e-mail: (ian@ece.gatech.edu).

\begin{tabular}{r|rrrrr} 
Set & $S_{T A}$ & Mosaic & $S_{T A L}$ & $N_{c}$ & $k$ \\
\hline \hline 1 & 1 & $T_{1}$ & 7 & 7 & 12 \\
\hline 2 & 3 & $M_{1}$ & 7 & 21 & 21 \\
\hline 3 & 7 & $T_{2}$ & 7 & 49 & 30 \\
\hline 4 & 12 & $M_{2}$ & 7 & 84 & 39 \\
\hline 5 & 19 & $T_{3}$ & 7 & 133 & 48 \\
\hline 6 & 27 & $M_{3}$ & 7 & 189 & 57
\end{tabular}

TABLE 3: Set of some scenarios when the shape of the TA follows some mosaic graph $M_{n}$ with $k=3(6 n+7)$ or $T_{n}$ with $k=6(3 n-1)$.

\section{A.1 Definition of $\mathrm{D}_{0}$ and $\mathrm{D}_{1}$}

In [33], [34] the name Phase-type Markov Renewal Process (PH-MRP) was used for the MAP. In a parallel way to [33], [34], here we also can refer to a Discrete PH-MRP (D-PH-MRP) for the D-MAP, where matrix $\mathbf{D}_{1}$ is a product of two matrices, $\mathbf{D}_{1}=\mathbf{D}_{1 a} \mathbf{D}_{1 b} . \mathbf{D}_{1 a}$ is a matrix of dimension $N_{c} \times k$ that contains transition probabilities from any of the $N_{c}$ transient states to any of the $k$ absorbing states and $\mathbf{D}_{1 b}$ a stochastic matrix of dimensions $k \times N_{c}$ that takes into account the transition probabilities from any of the $k$ absorbing states to any of the $N_{c}$ transient states. The absorbing states are also ephemeral since after each absorption an instantaneous restarting of the Markov process in any of the $N_{c}$ states occurs.

\section{A.1.1 Definition of matrices $\mathbf{D}_{0}$ and $\mathbf{D}_{1 a}$}

As illustrative example, when a TA contains three SCs, $S_{T A}=3$, and one TAL configured by a single TA, $S_{S T A}=1, N_{c}=3 \times 1=3$ and $k=9$. Matrix $\mathbf{D}_{0}$ is given by

$$
\mathbf{D}_{0}=\left[\begin{array}{ccc}
0 & P_{S E}(\alpha) & P_{S}(\alpha) \\
P_{N W}(\alpha) & 0 & P_{S W}(\alpha) \\
P_{N}(\alpha) & P_{N E}(\alpha) & 0
\end{array}\right]
$$

and $\mathbf{D}_{1 a}$ is shown in Figure 21 . 


$$
\mathbf{D}_{1 a}=\left[\begin{array}{ccccccccc}
0 & 0 & P_{N W}(\alpha) & 0 & P_{S W}(\alpha) & P_{N E}(\alpha) & 0 & P_{N}(\alpha) & 0 \\
P_{N E}(\alpha) & 0 & 0 & P_{S}(\alpha) & 0 & P_{N}(\alpha) & 0 & 0 & P_{S E}(\alpha) \\
0 & P_{S}(\alpha) & 0 & P_{S E}(\alpha) & P_{N W}(\alpha) & 0 & P_{S W}(\alpha) & 0 & 0
\end{array}\right] ;
$$

Fig. 21: Matrix $\mathbf{D}_{1 a}$ for $S_{T A}=3$ and $S_{T A L}=1$.

For other scenarios, matrices $\mathbf{D}_{0}=$ and $\mathbf{D}_{1 a}$ are easily derived based on geometric considerations. Hence, in Fig. 10 we have one TA composed of three SCs -a mosaic graph $M_{1}$, [24]- and one TAL composed of seven TAs, that is, $S_{T A}=3, S_{T A L}=7$, $N_{c}=3 \times 7=21$, and the number of SCs surrounding the TAL, $k=21$. Table 3 summarizes other configurations based on mosaic graphs $M_{n}$ and $T_{n}$

\section{A.1.2 Definition of $\mathbf{D}_{1 b}$ for the central policy}

It is implemented when the process restarts at the central TA of the new TAL. It is suited when the UEs moves according to a random-walk, in our model when $\alpha \approx 1$, see the routing probabilities of Table I. Hence, for the case when $S_{T A}=3$ and $S_{T A L}=1$, matrix $\mathbf{D}_{1 b}$ is defined, according to geometric considerations

$$
\mathbf{D}_{1 b}=\left[\begin{array}{l}
\mathbf{I}_{3} \\
\mathbf{I}_{3} \\
\mathbf{I}_{3}
\end{array}\right]
$$

where $\mathbf{I}_{3}$ is the identity matrix of size $3 \times 3$.

\section{A.1.3 Definition of $\mathbf{D}_{1 b}$ for the adaptive policy}

When the UEs do not follow a random-walk, common sense dictates that the central policy could be not the best solution, see the routing probabilities of table I. Here we define the adaptive TAL configuration which takes into account the residence time in the TAL. To that purpose, let us consider the matrix $\Delta$ with size $N_{c} \times k$ below. Its component $\Delta_{i, j}$ is the mean sojourn time of the Semi-Markov (SM) process in the TAL, i.e., in the set of transient states that conforms the TAL, under the conditions that initially the SM process starts in state $i$, i.e. in SC $i$, at $t=0^{+}$and leaves or abandons the TAL via the absorbing state $j$,

$$
\boldsymbol{\Delta}=\left[\mathbf{I}-\mathbf{D}_{0}\right]^{-2} \mathbf{D}_{1 a}
$$

In the example of Figure 10, $S_{T A}=3$ and $S_{T A L}=$ 7 , so $N_{c}=k=21$. Then, taking into account the initial state of the SM-process we have the following column vector,

$$
\boldsymbol{\delta}=\boldsymbol{\Delta} \mathbf{e}=\left[\mathbf{I}-\mathbf{D}_{0}\right]^{-2} \mathbf{D}_{1 a}=\left[\mathbf{I}-\mathbf{D}_{0}\right]^{-1} \mathbf{e}
$$

where the last equality follows from the fact that $\left[\mathbf{I}-\mathbf{D}_{0}\right]^{-1} \mathbf{D}_{1 a}=\mathbf{e}$. In (20), each element $\delta_{i}$ is the mean number of transitions performed by a UE before leaving the TAL, under the condition that the UE starts in state $i$ (SC $i$ ) at $t=0^{+}$. Therefore, the optimization consist in choosing the SC $i$ among a set $S_{T A}$ where $\delta_{i}$ achieves the highest value. In our example of Figure 10 it is necessary to find the maximum $\delta_{i}$ for the three groups of SCs, i.e., Group 0: $\{0,3,6,9,12,15,18\}$, Group 1: $\{1,4,7,10,13,16,19\}$ and Group 2: $\{2,5,8$, $11,14,17,20\}$. In other words, as an example, suppose the TAUs executed when the UE visits absorbing state 36. This state corresponds to the Group 1, see Figure 10(a) and 10(b). Then the starting SC $i$ in the new TAL assigned to the UE will be one of the Group 1, i.e, $i=k$ such that $\delta_{k}>\delta_{j}$ for $\forall j \neq k$. In case of a tie, the assignment of the initial SC $i$ is done by flipping a coin. Due to the lack of space we omit the details for the corresponding matrix $\mathbf{D}_{1 b}$.

\section{A.2 On the number of labeled transitions}

First we are interested in the number of labeled transitions among a total of $z$ transitions of the DTMC. Let $\mathbf{M}^{(z)}=\left[M_{0}^{(z)}, M_{1}^{(z)}, \ldots, M_{N_{c}-1}^{(z)}\right]^{t}$ denote a column vector, being $M_{k}^{(z)}$ the mean number of labeled transitions, equivalently TAUs, assuming that the initial position of the process, of the UE, is at cell $k$, $0 \leq k \leq N_{c}-1 . N_{c}$ is the total number of states, equivalently, the number of SCs in the TAL. Then, we can write the following recursion,

$$
\mathbf{M}^{(z)}=\left\{\begin{array}{l}
\mathbf{0}, \quad z=0 \\
\mathbf{D}_{1} \mathbf{e}, \quad z=1 \\
\mathbf{D}_{0} \mathbf{M}^{(z-1)}+\mathbf{D}_{1}\left[\mathbf{e}+\mathbf{M}^{(z-1)}\right], \quad z>1
\end{array}\right.
$$

Taking into account (18), expression can be written as

$$
\mathbf{M}^{(z)}=\left\{\begin{array}{l}
\mathbf{0}, \quad z=0 \\
\sum_{m=0}^{z-1} \boldsymbol{\Pi}^{m} \mathbf{D}_{1} \mathbf{e}, \quad z \geq 1
\end{array}\right.
$$

Second, we want to observe the process during a time interval defined by a random variable exponentially distributed, with rate $\lambda_{c}$. In fact, the observable exponential time is coincident with the inter-arrival time of incoming calls to our UE, that is, we assume a Poisson arrival process and the PASTA (Poisson 
Arrival See Time Averages)property, can be applied, [31]. We assume that the sojourn time in any SC is characterized by the density function $f_{m}(t)$, with Laplace Transform (LT) $f_{m}^{*}(s)$, which means that we are in presence of a Semi-Markov process. If $\boldsymbol{\pi}_{v}=$ $\left[\pi_{v, 0}, \pi_{v, 1}, \ldots, \pi_{v, N_{c}-1}\right]$ is the stochastic vector solution of $\boldsymbol{\pi}_{v}=\boldsymbol{\pi}_{v} \boldsymbol{\Pi}$, with $\boldsymbol{\pi}_{v} \boldsymbol{e}=1$, then, $\pi_{v, k}$ represents the percentage of visits to state $k$. Since $f_{m}(t)$ is the same for all SCs, $\pi_{v, k}$ equals the percentage of time the system is visiting state $k, p_{k}$, i.e., $p_{k}=\pi_{v, k}$, in vector notation $\mathbf{p}=\boldsymbol{\pi}_{v}$.

Then, since the interval starts at random point in the time axis - Poisson arrival process-, the probability to register a total of $z$ transitions (labeled plus unlabeled) during the observable exponential time, $\beta(z)$, is given by, [7],

$$
\beta(z)=\left\{\begin{array}{l}
1-f_{m, r}^{*}\left(\lambda_{c}\right), \quad \text { for } z=0 \\
f_{m, r}^{*}\left(\lambda_{c}\right)\left[1-f_{m}^{*}\left(\lambda_{c}\right)\right]\left[f_{m}^{*}\left(\lambda_{c}\right)\right]^{z-1} \\
\quad \text { for } z>0
\end{array}\right.
$$

being $f_{m, r}^{*}(s)$ the LT of the residual life time in a given state,

$$
f_{m, r}^{*}(s)=\lambda_{m} \frac{1-f_{m}^{*}(s)}{s}
$$

Hence, for instance, if $f_{m}(t)$ obeys to a Gamma distribution [35], expression (1), we have:

$$
\begin{array}{r}
f_{m}^{*}\left(\lambda_{c}\right)=\left(\frac{\lambda_{m} \gamma}{\lambda_{c}+\lambda_{m} \gamma}\right)^{\gamma}=\left(\frac{\gamma}{\theta+\gamma}\right)^{\gamma}, \\
f_{m, r}^{*}\left(\lambda_{c}\right)=\frac{1-f_{m}^{*}\left(\lambda_{c}\right)}{\theta}, \quad \theta=\lambda_{c} / \lambda_{m} .
\end{array}
$$

Then, unconditioning (22) with respect to (23) we get

$$
\begin{aligned}
\mathbf{M}= & {\left[M_{0}, M_{1}, \ldots, M_{N_{c}-1}\right]^{t} } \\
& =\sum_{z=0}^{\infty} \beta(z) \mathbf{M}^{(z)} \mathbf{e} \\
& \left.=f_{m, r}^{*}\left(\lambda_{c}\right)\right]\left[\mathbf{I}-f_{m}^{*}\left(\lambda_{c}\right) \mathbf{\Pi}\right]^{-1} \mathbf{D}_{1} \mathbf{e},
\end{aligned}
$$

In (26), $M_{i}$ is the mean number of labeled transitions registered during the observable exponential time, conditioned to the fact that at the starting instant of the observation interval, the process is in state $i$, i.e., in the SC $i$.

\section{A.3 Actions performed at the arrival of an incom- ing call}

When an incoming call is successfully delivered to the UE and no new TAL is assigned (that is, the UE stays in the same TAL), we can assert that at the starting instant of the observation interval the process is in state $i$ with probability $p_{i}$, where $p_{i}$ is the $i$-th component of the stochastic vector $\mathbf{p}$ solution to $\mathbf{p}=\boldsymbol{\pi}_{v}=\boldsymbol{\pi}_{v} \boldsymbol{\Pi}$, as it has been said before. Therefore, in this case, the unconditioned mean number of labeled transitions between two consecutive call arrivals is

$$
\left.\overline{\text { \#Labeled }}=\mathbf{p} f_{m, r}^{*}\left(\lambda_{c}\right)\right]\left[\mathbf{I}-f_{m}^{*}\left(\lambda_{c}\right) \mathbf{\Pi}\right]^{-1} \mathbf{D}_{1} \mathbf{e} .
$$

At the arrival of an incoming call, the UE can be found in a SC that does not necessarily belongs to the TA located at the center of the current TAL. If this is the case, and thinking on the random-walk mobility model, it would be convenient to perform the central policy configuration just like after a TAL crossing; that is, to provide a new TAL to the UE after an incoming call, such that the SC where the UE is currently found belongs to the TA located at the center of the new TAL. In this case, the initial vector $\mathbf{p}$ can not be used in equation (27). Instead, we have to proceed according to the next lines.

First, we define two row vectors, $\mathbf{P}_{f s c}=$ $\left[P_{f s c, 0}, P_{f s c, 1}, \ldots, P_{f s c, N_{c}-1}\right] \quad$ and $\quad \mathbf{P}_{i s c}=$ $\left[P_{i s c, 0}, P_{i s c, 1}, \ldots, P_{i s c, N_{c}-1}\right] . \quad P_{f s c, k}$ denotes the probability that an arbitrary incoming call finds the UE visiting cell $k, k \in\left[0, N_{c}-1\right] . P_{i s c, j}$ is the probability that just immediately after the reception of the last incoming call, the UE be positioned in cell $j, j \in\left[0, N_{c}-1\right]$. In general, and thinking about the central policy, $\mathbf{P}_{i s c} \neq \mathbf{P}_{\text {fsc }}$. Both stochastic vectors can be obtained as follows. Let us define $\operatorname{Pr}(k / j)$ as the conditional probability that the next incoming call will find the UE visiting SC $k$, conditioned to the fact that just immediately after the reception of the last incoming call the UE was positioned in SC $j$. Then, the following relationship is satisfied

$$
\mathbf{P}_{f s c}=\mathbf{P}_{i s c} \mathbf{T}
$$

where $\mathbf{T}$ is

$$
\mathbf{T}=\left[\begin{array}{ccc}
\operatorname{Pr}(0 / 0) & \ldots & \operatorname{Pr}\left(N_{c}-1 / 0\right) \\
\operatorname{Pr}(0 / 1) & \ldots & \operatorname{Pr}\left(N_{c}-1 / 1\right) \\
\vdots & \ddots & \vdots \\
\operatorname{Pr}\left(0 / N_{c}-1\right) & \ldots & \operatorname{Pr}\left(N_{c}-1 / N_{c}-1\right)
\end{array}\right]
$$

and obeys the expression

$$
\begin{aligned}
& \mathbf{T}=\sum_{z=0}^{\infty} \beta(z) \mathbf{\Pi}^{z} \\
=[1 & \left.-f_{m, r}^{*}\left(\lambda_{c}\right)\right] \mathbf{I} \\
& \quad+f_{m, r}^{*}\left(\lambda_{c}\right)\left[1-f_{m}^{*}\left(\lambda_{c}\right)\right] \mathbf{\Pi}\left[\mathbf{I}-f_{m}^{*}\left(\lambda_{c}\right) \mathbf{\Pi}\right]^{-1}(30)
\end{aligned}
$$


On the other hand, because of the centering process immediately after the successful reception of an incoming call, we can write,

$$
\mathbf{P}_{i s c}=\mathbf{P}_{f s c} \mathbf{I}_{m d f} .
$$

In (31) the matrix $\mathbf{I}_{m d f}$ is the identity matrix modified, and takes into account the centering process immediately after the successful reception of an incoming call to the UE. For instance, for the TAL of Figure 10(b), we have,

$$
\mathbf{I}_{m d f}=\left[\begin{array}{lllllll}
\mathbf{I}_{3} & \mathbf{0}_{3} & \mathbf{0}_{3} & \mathbf{0}_{3} & \mathbf{0}_{3} & \mathbf{0}_{3} & \mathbf{0}_{3} \\
\mathbf{I}_{3} & \mathbf{0}_{3} & \mathbf{0}_{3} & \mathbf{0}_{3} & \mathbf{0}_{3} & \mathbf{0}_{3} & \mathbf{0}_{3} \\
\mathbf{I}_{3} & \mathbf{0}_{3} & \mathbf{0}_{3} & \mathbf{0}_{3} & \mathbf{0}_{3} & \mathbf{0}_{3} & \mathbf{0}_{3} \\
\mathbf{I}_{3} & \mathbf{0}_{3} & \mathbf{0}_{3} & \mathbf{0}_{3} & \mathbf{0}_{3} & \mathbf{0}_{3} & \mathbf{0}_{3} \\
\mathbf{I}_{3} & \mathbf{0}_{3} & \mathbf{0}_{3} & \mathbf{0}_{3} & \mathbf{0}_{3} & \mathbf{0}_{3} & \mathbf{0}_{3} \\
\mathbf{I}_{3} & \mathbf{0}_{3} & \mathbf{0}_{3} & \mathbf{0}_{3} & \mathbf{0}_{3} & \mathbf{0}_{3} & \mathbf{0}_{3} \\
\mathbf{I}_{3} & \mathbf{0}_{3} & \mathbf{0}_{3} & \mathbf{0}_{3} & \mathbf{0}_{3} & \mathbf{0}_{3} & \mathbf{0}_{3}
\end{array}\right]
$$

where $\mathbf{I}_{3}$ is the identity matrix of size $3 \times 3$ and $\mathbf{0}_{3}$ is a zeros matrix of size $3 \times 3$. As a particular case, when no centering is executed after each incoming call to the UE, then $\mathbf{I}_{m d f}=\mathbf{I}$.

Inserting (28) into (31) we get

$$
\mathbf{P}_{i s c}=\mathbf{P}_{i s c} \mathbf{T I}_{m d f}
$$

Notice that in (33) $\mathbf{T I}_{m d f}$ is an stochastic matrix, that is $\mathbf{T I} \mathbf{I}_{m d f} \mathbf{e}=\mathbf{T e}=\mathbf{e}$. Then, solving (33) with the normalization condition $\mathbf{P}_{i s c} \mathbf{e}=1$ we obtain the $\mathbf{P}_{i s c}$ and then from (28) we get $\mathbf{P}_{f s c}$.

Therefore, in the case of central policy or adaptive policy we will use in (27) the probabilities given by $\mathbf{P}_{i s c}$ instead of $\mathbf{p}$. 